\title{
TALEN-based chemically inducible, dimerization-dependent, sequence-specific nucleases
}

\author{
Daisuke Matsumoto ${ }^{1, \dagger}$, Hirokazu Tamamura ${ }^{1}$, and Wataru Nomura ${ }^{1, \dot{+}, *}$ \\ ${ }^{1}$ Institute of Biomaterials and Bioengineering, Tokyo Medical and Dental University, 2- \\ 3-10 Kandasurugadai, Chiyoda-ku, Tokyo, 101-0062, Japan
}

*To whom correspondence should be addressed at Graduate School of Biomedical and Health Sciences, Hiroshima University, 1-2-3 Kasumi, Minami-ku, Hiroshima 734-8553, Japan; Tel: +81-82-257-8553; Fax: +81-82-257-5309; E-mail: wnomura@,hiroshimau.ac.jp

${ }^{\dagger}$ Current affiliation: Department of Chemistry, The Scripps Research Institute, Torrey Pines Road, La Jolla, CA 92037, USA

$\$$ Current affiliation: Graduate School of Biomedical and Health Sciences, Hiroshima

University, 1-2-3 Kasumi Minami-ku, Hiroshima, 734-8553, Japan 


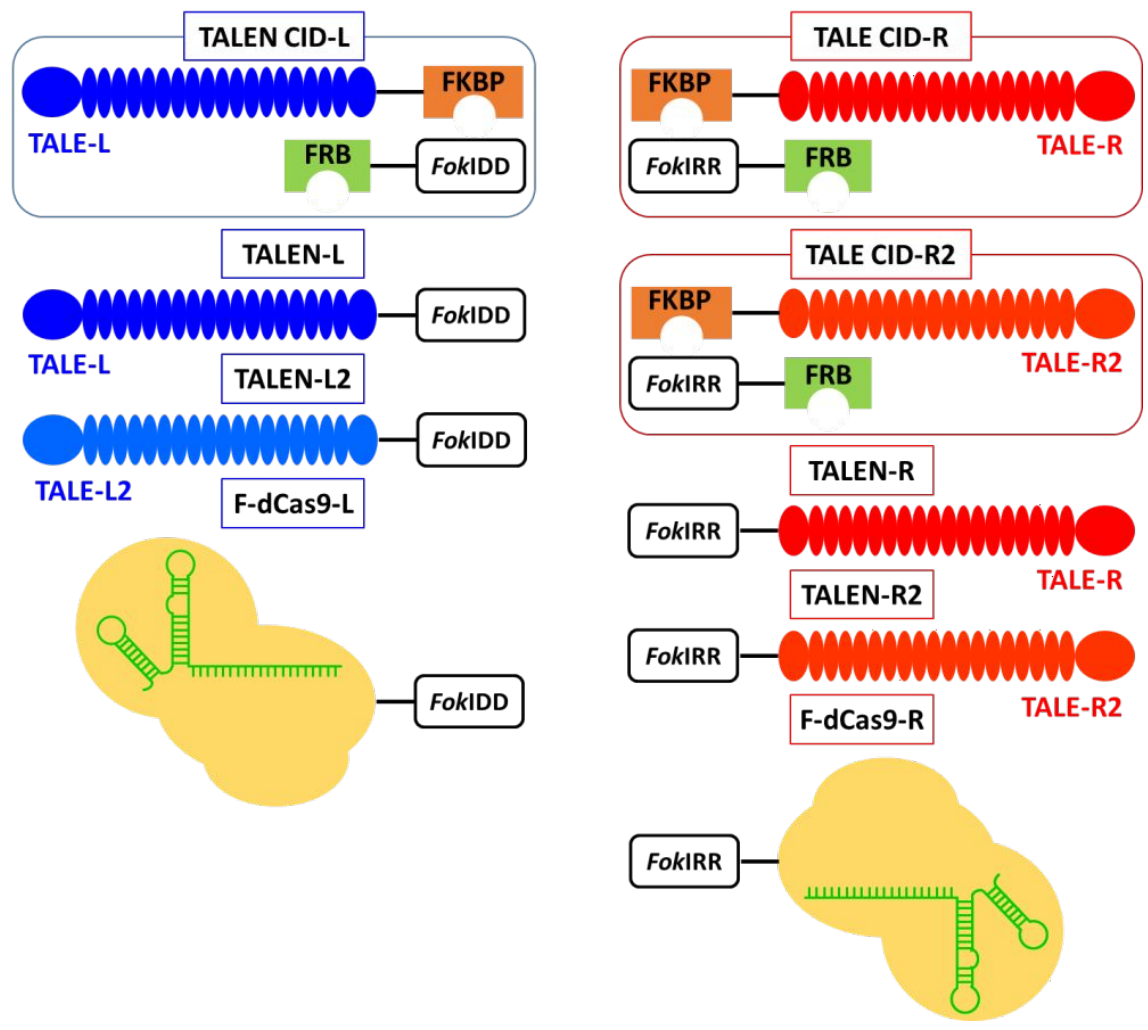

\begin{tabular}{|c|c|l|}
\hline AAVS1 L-arm & AAVS1 R-arm & experiments (Figure\#) \\
\hline & & \\
\hline TALEN-L & TALEN-R & 2C, 2D \\
\hline TALEN-L & TALEN CID-R & 2C, 2D, 4A*,4B \\
\hline TALEN CID-L & TALEN-R & 2C, 2D \\
\hline TALEN-L & F-dCas9-R & $3 \mathrm{~B}$ \\
\hline TALEN-L2 & F-dCas9-R & $3 \mathrm{~B}$ \\
\hline F-dCas9-L & TALEN-R2 & $3 \mathrm{~B}$ \\
\hline F-dCas9-L & TALEN CID-R2 & $3 C^{*}$ \\
\hline
\end{tabular}

*Different linker length between FRB and FokIDD was also tested.

Figure S1. Summary of components of TALE or dCas9 based nucleases used in the manuscript. Combinations of intact or chemically-inducible nucleases are also described. 


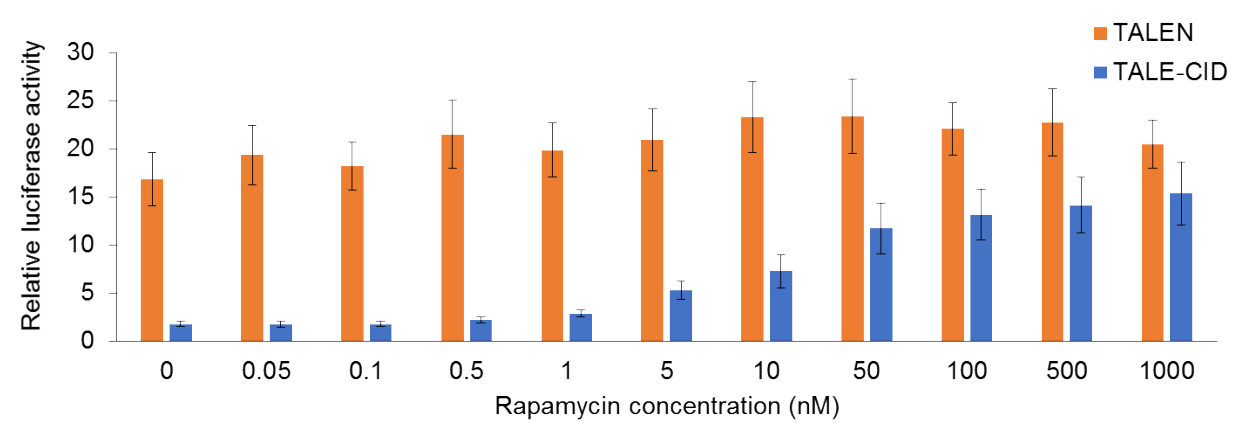

Figure S2. Relation between rapamycin concentration and activity. Each plasmid DNA was transfected into 293A cells. For TALE-CID, TALEL-FokIDD, TALE-R-FKBP, and FRB-(GGGGS) $)_{3}$-FokIRR. At $24 \mathrm{~h}$ after transfection, rapamycin induction was performed under the concentration of $0,0.05,0.1,0.5,1,5,10,50,100,500,1000 \mathrm{nM}$. Luciferase activity was detected the same way as previous result. Error bar shows S.E.M.

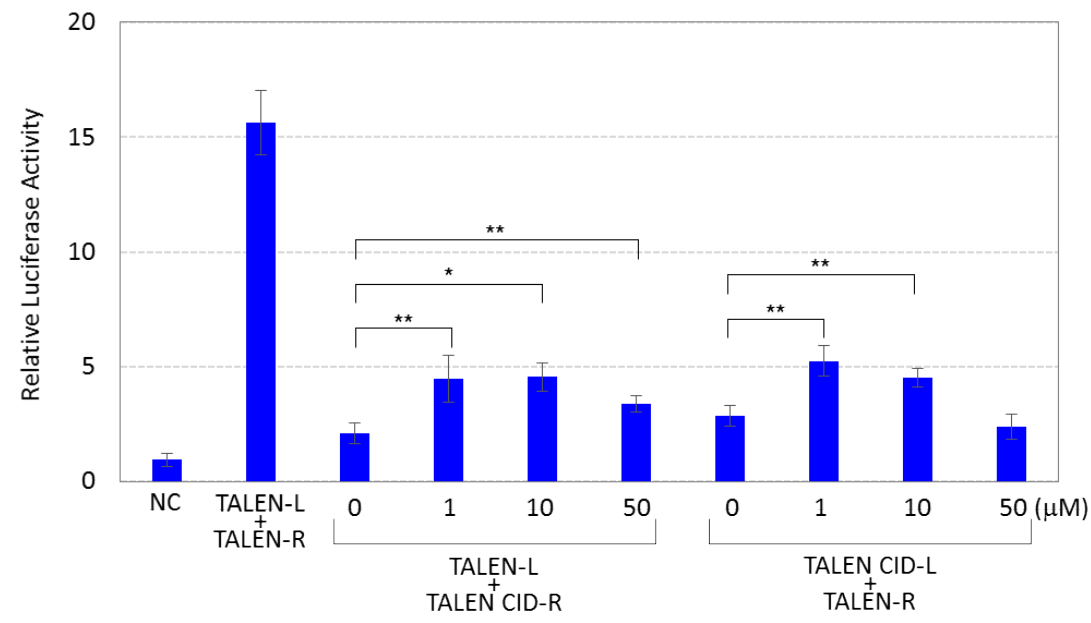

Figure S3. Effects of rapamycin concentration on induced cleavage activity. Data are expressed as means \pm S.D. of four replicate experiments. Two-sided Student's $t$-tests were performed to assess the significance of differences between the control ( $0 \mu \mathrm{M}$ rapamycin) and each rapamycin concentration. $* \mathrm{P}<0.01 .{ }^{*} \mathrm{P}<0.005$. 


\section{(A)}

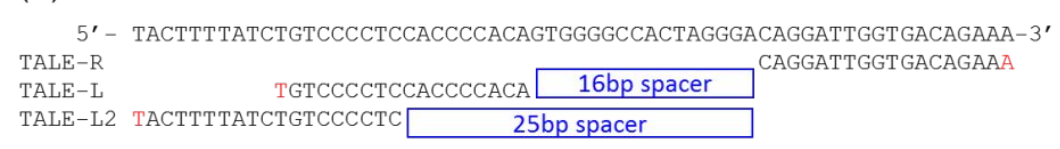

(B)

(C)
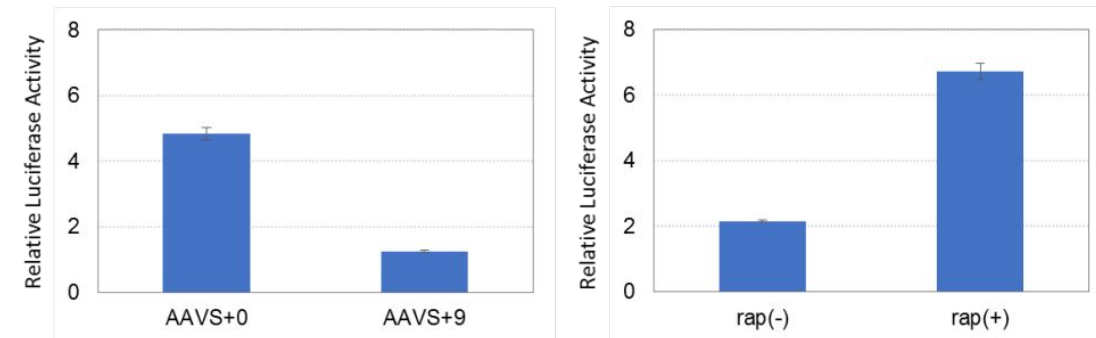

Figure S4. SSA assays for detection of DNA cleavage in cultured cells by using TALENL2. (A) Target sequences for TALE-R, -L and -L2. Length of spacer sequences are indicated. (B) DNA cleavage for AAVS and AAVS+9 sequence by TALEN-L2 and TALEN-R pair. (C) Rapamycin induced DNA cleavage by TALEN-L2 and TALEN CID$\mathrm{R}$ pair for AAVS sequence. Data are expressed as means \pm S.E.M. of three replicate experiments.

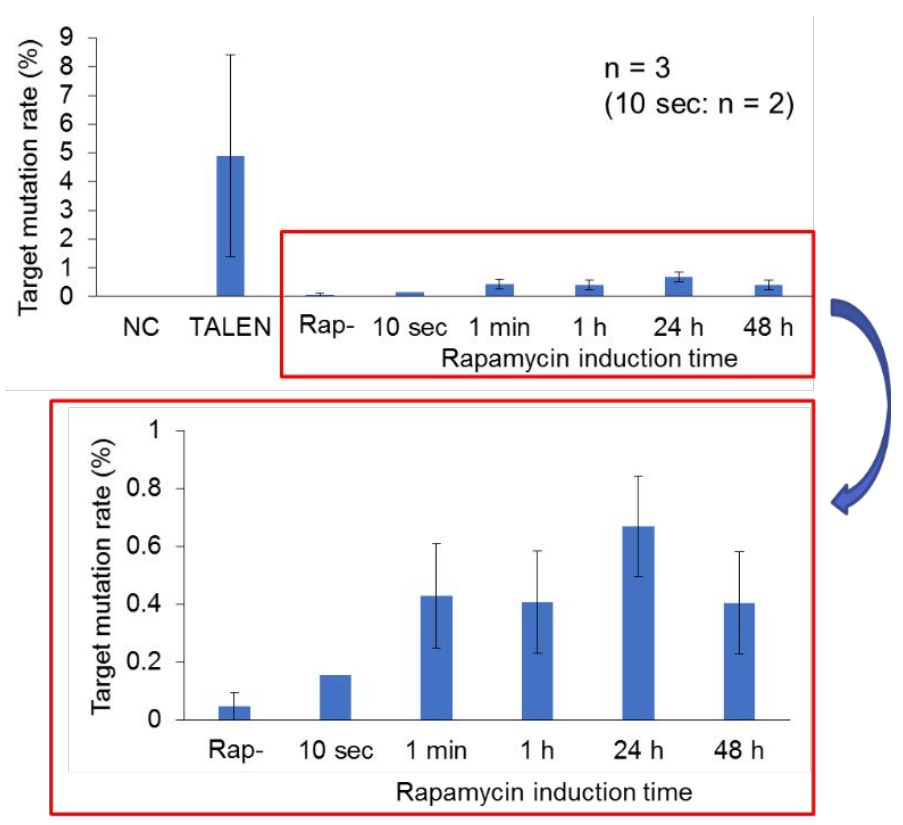

Figure S5. Results of genomic DNA cleavage by short-term rapamycin addition. 293A cells were transfected each nuclease plasmid DNAs by electroporation. Cells were incubated under $1 \mu \mathrm{M}$ rapamycin for each time ( $10 \mathrm{sec}, 1 \mathrm{~min}, 1 \mathrm{~h}, 24 \mathrm{~h}$, and $48 \mathrm{~h}$ ) at $\mathrm{CO}_{2}$ incubator. Cells in short induction sample were rinsed by DMEM three times and incubated in DMEM without rapamycin until $48 \mathrm{~h}$ after induction. Mutation was detected by T7E1 assay. Error bar shows S.E.M. $n=2$ (10 sec sample). $n=3$ (1 min, $1 \mathrm{~h}, 24 \mathrm{~h}$, and $48 \mathrm{~h})$. 
Table S1. Primer sequences.

\begin{tabular}{|c|c|}
\hline Name & Sequence $\left(5^{\prime}\right.$ to $\left.3^{\prime}\right)$ \\
\hline $3 \times F L A G+N L S$ & GACTACAAAGACCATGACGGTGATTATAAAGATCATGACATCGATTAC \\
\hline code & AAGGATGACGATGACAAGGGAGATCCAAAAAA \\
\hline $3 \times F L A G+N L S$ & TTTCCTTTTTTCTTGGGATCCACCTTGCGTTTCTTTTTCGGGTCAAC \\
\hline complement & СTTTCTCTTCTTTTTTGGATCTCCCTTGTCAT \\
\hline $\begin{array}{l}\text { flagNLScode } \\
\text { subF }\end{array}$ & AAGAGGAGGCTAGCGCCACCATGGACTACAAAGACCATGACGG \\
\hline $\begin{array}{l}3 \times f l a g N L S S u b R \\
2\end{array}$ & 'TTCTCCTCCTCGTTTAAACTGTTGTTCTAGATCCACCTACTTTCC \\
\hline $\begin{array}{l}\text { TALEN- } \\
\text { N_XbalFw }\end{array}$ & AAGAGGAGTCTAGAGTAGATTTGAGAACTTTGGGATATTCAC \\
\hline $\begin{array}{l}\text { TALEN- } \\
\text { C_BamHIRv }\end{array}$ & CCTCTTCTGGATCCTGCCACTCGATGTGATGTCC \\
\hline D10A_Fw & AAGAAGTACTCCATTGGGCTCGCTATCGGCACAAACAGC \\
\hline D10A_Rv & CGAGCCCAATGGAGTACTTCTTGTCCATGGTGGCAAG \\
\hline H840A_Fw & CCGACTACGACGTGGATGCTATCGTGCCCCAGTC \\
\hline H840A_Rv & GCATCCACGTCGTAGTCGGAGAGCCGATTGATGTCC \\
\hline $\begin{array}{l}\text { GlySilentMut } \\
\text { Fw }\end{array}$ & GGCTGTCAAGAAAACTGATCAATGGAATCCGAGACAAGCAGAGTGG \\
\hline $\begin{array}{l}\text { GlySilentMut } \\
\text { Rv }\end{array}$ & TCCATTGATCAGTTTTCTTGACAGCCGCCCCCATCCTGTATATCGG \\
\hline Xbal hCas9 Fw & GGAGAAGATCTAGAGACAAGAAGTACTCCATTGGGC \\
\hline $\begin{array}{l}\text { hCas9 BamHI } \\
\text { Rv }\end{array}$ & IICTCTTCTGGATCCCACCTTCCTCTTCTTCTTGGG \\
\hline $\begin{array}{l}\text { EcoRI_LucC_F } \\
\text { w }\end{array}$ & CСTCGAATTCCGACATTTATAATGAACGTGAATTGCTC \\
\hline EBV Rv primer & GTGGTTTGTCCAAАCTCATC \\
\hline LucN_STOP_A & GGAGTCTAGAGAGGAATTCTTTTTTTCTGTCACCAATCCTGTCCCTAG \\
\hline AVS1_EcoRI_R & TGGCCCCACTGTGGGGTGGAGGGGACAGTCACATAGGACCTCTCACAC \\
\hline $\mathrm{v}$ & ACAG \\
\hline
\end{tabular}




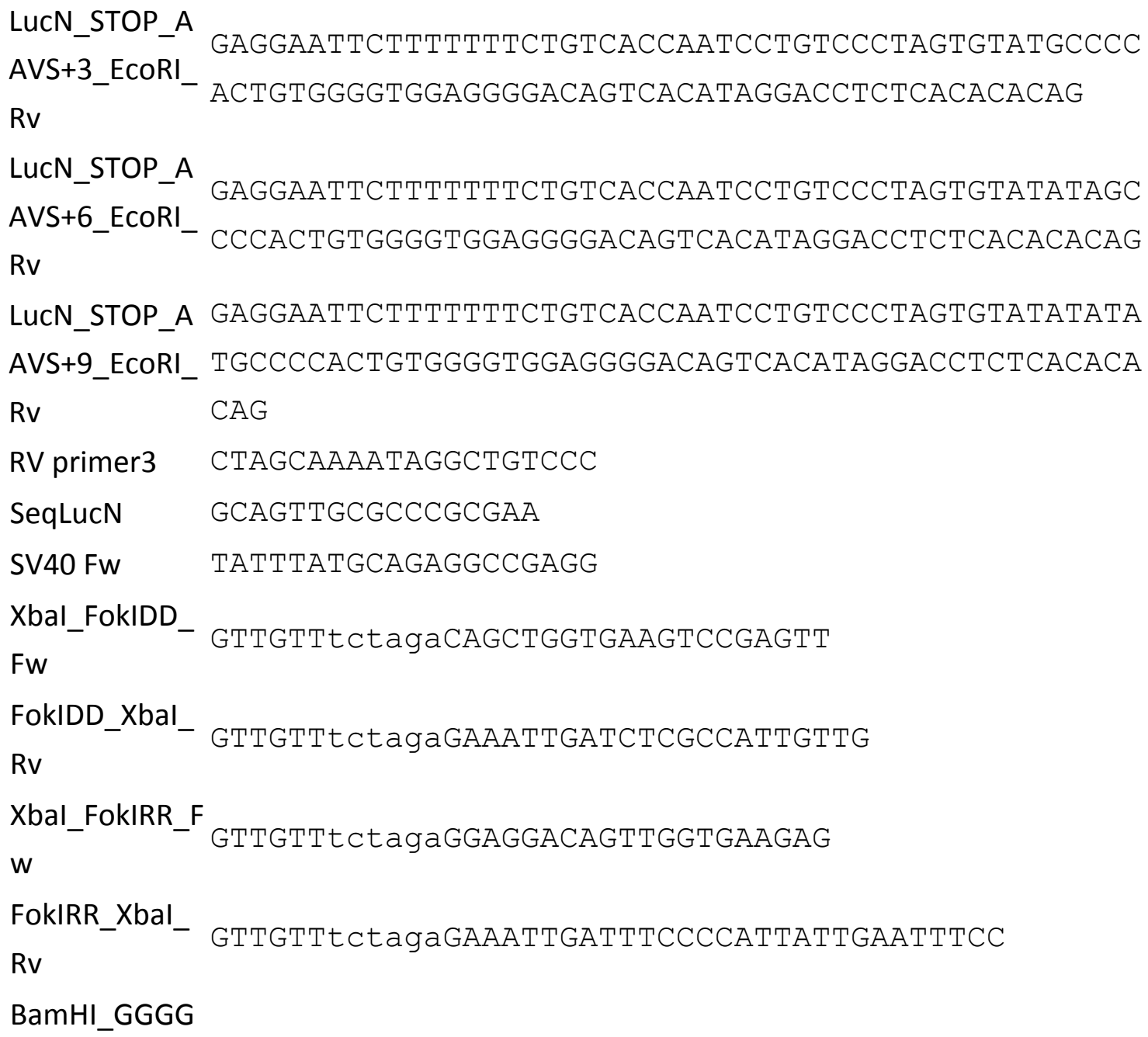


linker_BamHI_

Rv

BamHI_GGGG

S5-

linker_BamHI

GTTGTTGGATCCGGTGGCGGTGGCTCCGGTGGCGGTGGCTCCGGTGGC

Fw

GGTGGCTCCGGTGGCGGTGGCTCCGGTGGCGGTGGCTCCGGATCCGTT GTT

BamHI_GGGG

S5-

AACAACGGATCCGGAGCCACCGCCACCGGAGCCACCGCCACCGGAGCC linker_BamHI ACCGCCACCGGAGCCACCGCCACCGGAGCCACCGCCACCGGATCCAAC Rv

AAVS1-

T7E1_Fw

GATCAGTGAAACGCACCAGA

AAVS1-

T7E1_Rv

CCCTGTCATGGCATCTTCCAG 